\title{
Eficiencia anual en una operación comercial de ceba final de bovinos con la tecnología de silvopastoreo
}

\author{
Guevara Viera, R.V. ${ }^{\circledR}$; Lascano Armas, P.J. ${ }^{2}$; Arcos Álvarez, C.N. ${ }^{2}$; Toro Ramírez, A. del'; Peña del Río, E.R. ${ }^{4}$; Curbelo \\ Rodríguez, L.M. ${ }^{3}$; Guevara Viera, G.E. ${ }^{1}$; Serpa García, G. ${ }^{1}$ y Soto Senra, S.A. ${ }^{3}$
}

'Facultad de Ciencias Agropecuarias. Campus de Yanuncay. Universidad de Cuenca. Cuenca. República de Ecuador.

${ }^{2}$ Unidad Académica de Ciencias Agropecuarias y Recursos Naturales (UA-CAREN). Carrera de Medicina Veterinaria. Universidad Técnica de Cotopaxi. Latacunga. Ecuador.

${ }^{3}$ Centro de estudios para el desarrollo de la producción animal (CEDEPA). Facultad de Ciencias Agropecuarias. Universidad de Camagüey. Cuba de ceba. Municipio Guáimaro. Camagüey. Cuba.

${ }^{4}$ Empresa Pecuaria Rectángulo de ceba. Municipio Guáimaro. Camagüey. Cuba.

\section{PALABRAS CLAVE ADICIONALES}

Rumiantes.

Pastoreo.

Rentabilidad.

Factores de manejo.
Ganancia de peso.

\section{RESUMEN}

Con el objetivo de evaluar factores que afectan la eficiencia en una asociación de Leucaena leucocephala 'Perú' con pastos para ceba final de bovinos, se analizaron desde el 2002 al 2012, 22 ciclos de ceba de toros Cebú en la unidad Represa \# 1 de la empresa pecuaria Rectángulo de ceba en Camagüey, Cuba. El suelo es pardo sin carbonatos. El clima es tropical húmedo de sabana (Aw) con precipitación media anual de $1183 \mathrm{~mm}$. Los factores evaluados fueron: la carga animal ( $\mathrm{a} / \mathrm{ha}$ ) y los años de evaluación. Se determinaron los cambios en el porcentaje de la población de pastos y de Leucaena por conteo de plantas. Se analizaron índices de ganancia diaria de peso, gastos e ingresos por operación. Leucaena 'Perú' alcanzó valores finales del $93 \%(p<0,05)$ en su población, con incremento de las poblaciones de Guinea común y otros pastos. Se alcanzaron ganancias elevadas en la carga mas baja $(p<0,05)$ con $0,981 \mathrm{~kg} / \mathrm{a} / \mathrm{d}$. La tecnología de silvopastoreo con la asociación de Leucaena leucocephala 'Perú' -gramíneas en secano, permitió obtener ganancias medias diarias de peso por encima de $0,800 \mathrm{~kg} / \mathrm{a} / \mathrm{d}$ en toros de ceba final que se mantuvieron por diez años con resultados económicos muy adecuados a esa tecnología y el nivel de insumos aplicados.

\section{Annual efficiency of a commercial finishing operation of fattening of cattle with silvopastoral technology}

\author{
SUMMARY
}

\section{ADDITIONAL KEYWORDS}

Ruminants.

Gain of weight.

Grazing.

Profitability.

Management.

\section{INFORMACIÓN}

\section{Cronología del artículo.}

Recibido/Received: 23.06 .2015

Aceptado/Accepted: 27.01.2016

On-line: 11.06 .2016

Correspondencia a los autores/Contact e-mail:

rguevaraviera@yahoo.es

\section{INTRODUCCIÓN}

Los sistemas pecuarios con gramíneas y leguminosas arbustivas en asociación han aportado resultados
With the objective to evaluate the factors that affect performance related to the association of a mixture of pasture and Leucaena leucocephala 'Perú' for the end of cattle fattening, 22 fattening cycles were assessed in zebu bulls at the Represa \# 1 unit of Rectángulo de ceba in Camagüey, Cuba, from 2002 to 2012 . Soil is brown without carbonates. The climate is savannah tropical wet (Aw) with $1183 \mathrm{~mm}$ of annual rainfall. The factors evaluated were: stocking rate (a/ha) and years assessed. The changes in the percentage of the pasture and Leucaena populations were recorded through plant counting. Daily weight gain indexes, income and expenses per operation were assessed. Leucaena 'Perú' reached final values of $93 \%(p<0.05)$ in its population, with an increase in the populations of Guinea grass and other pastures. High income was reached for the lowest charge $(p<0.05)$ with $0.981 \mathrm{~kg} / \mathrm{a} / \mathrm{d}$. Silvopastoral technology with the association of Leucaena leucocephala 'Perú'-dryland grasses, allowed to obtain average daily weight gains over $0.800 \mathrm{~kg} / \mathrm{a} / \mathrm{d}$ at the end of the fattening of bulls which were held for ten years of evaluations with very suitable economic results according to such technology and the inputs levels applied. meritorios para una ganadería más congruente con el ambiente, logran elevar los ingresos de los ganaderos y contribuyen al desarrollo armónico de las zonas rurales (Guevara et al., 2009; Pérez Infante, 2010; Alonso, 
2011; Milera, 2013). En este contexto, la renovación de los pastizales, unido a la incorporación estratégica de plantas arbóreas-arbustivas en las áreas de pastoreo para operaciones comerciales de ceba final en pastoreo, se presenta como una alternativa tecnológica que contribuirá a mejorar la producción de esta categoría bovina.

En este sentido, el objetivo del estudio fue evaluar el efecto de la carga animal y los años en la dinámica en el tiempo de los indicadores de población de pastos, y la eficiencia productiva y económica en una asociación de Leucaena leucocephala 'Perú' con pastos mejorados y nativos para ceba vacuna final.

\section{MATERIALES Y MÉTODOS}

Este trabajo se realizó entre los años 2002-2012 en la unidad Represa \# 1, de la empresa pecuaria Rectángulo de Guáimaro en Camagüey, Cuba. El suelo es pardo sin carbonatos. El clima es tropical húmedo de sabana (Aw) y precipitación media anual de $1183 \mathrm{~mm}$ (71\% en el periodo lluvias).

El establecimiento de las leguminosa Leucaena leucocephala 'Perú', se llevó a cabo con pastoreo a fondo del área previo a la roturación en franjas de 2,0 $\mathrm{m}$ de ancho y 3,0 m de separación y siembra con 6,0 kg/ha de semilla inoculada de la leguminosa. Se consideró establecida, cuando las plantas alcanzaron 1,2 $\mathrm{m}$ de altura. El área se dividió en 20 cuartones. El tiempo de reposo en lluvia de 38-57 días y en seca de 57-76 días y ocupación de 2-4 días. Se suplementó con Norgold $®$ en dosis de $0,4-1,0 \mathrm{~kg} / \mathrm{a} / \mathrm{d}$.

Se analizaron 22 ciclos de ceba final en pastoreo de toros Cebú desde 2002 a 2012 (10 años). Se midió el efecto de la carga animal (a/ha) y los años evaluados. Se analizó la ganancia diaria de peso, peso final, gastos, costos e ingresos finales por operación. Se determinaron los\% de población inicial y final de las especies pratenses y la Leucaena por conteo de plantas / cuartón anualmente. Se realizó un análisis de varianza y prueba de Tukey, con el programa SYSTAT versión 7.0.

\section{RESULTADOS Y DISCUSIÓN}

En la tabla I, se muestra el efecto de la carga animal en las ganancias de peso y otros índices para la com- paración entre los rangos de este indicador estudiado (1,4-1,9 a/ha y 2,0-2,4 a/ha), donde se encontraron diferencias $(\mathrm{p}<0,05)$ a favor del menor rango con valores de $0,981 \mathrm{~kg} / \mathrm{a} / \mathrm{d}$ con una diferencia favorable de 0,232 $\mathrm{kg}$ respecto a la carga mayor.

Estos efectos se encontraron por Cino et al. (2011); Díaz et al. (2012) y Milera et al. (2013) para distintos escenarios ganaderos de las provincias de Camagüey, Habana, Granma y Matanzas en Cuba, donde se evaluaron en condiciones comerciales asociaciones de gramíneas-Leucaena y otras leguminosas, que presentaron incrementos de su población en el tiempo y que coincidió con una evolución anual bio-económica positiva de la tecnología de silvopastoreo y del sistema de ceba bovina (tabla II) y las ganancias de peso presentaron diferencias significativas $(\mathrm{p}<0,05)$ a favor de los años 6 al 10, con igual tendencia en los índices económicos, lo cual es un indicativo de gran estabilidad del sistema y también de su resiliencia en términos de productividad, aportes nutricionales, persistencia en la seca con un incremento en las poblaciones de la arbustiva con baja suplementación (Milera, 2013; Cino et al., 2011; Ku Vera, 2014).

\section{CONCLUSIONES}

El incremento de la carga animal, aunque afecta en modo significativo $(\mathrm{p}<0,05)$ la ganancia de peso diaria de los animales y los ingresos monetarios finales respecto a cargas menores a 2,0 animales/ha, logra mantener valores cercanos a $0,759 \mathrm{~kg} / \mathrm{a} / \mathrm{d}$ y mejora la persistencia en el tiempo de los componentes Leucaena 'Perú' y Guinea 'Común', que incrementaron $(p<0,05)$ sensiblemente sus poblaciones en el pastizal y permitió obtener ganancias medias diarias de peso por encima de $0,800 \mathrm{~kg} / \mathrm{a} / \mathrm{d}$ en toros de ceba final que se mantuvieron por diez años con resultados económicos muy adecuados.

\section{AGRADECIMIENTOS}

Se agradece a la Empresa pecuaria Rectángulo de ceba de Camagüey, Cuba por los recursos aportados para este trabajo y permitir el acceso a las áreas de la finca y el monitoreo de las operaciones de ceba, como parte de este proyecto sobre ganado de carne que ejecutaron la Universidad de Camagüey, Cuba y el CITMA en Camagüey y en el que participaron además estudiantes de posgrado de la Universidad de Cotopaxi en Ecuador.

Tabla I. Efecto de la carga animal en la ganancia de peso, peso final, gastos por suplementos e ingresos finales por operaciones en sistemas de ceba bovina en silvopastoreo con Leucaena 'Perú' asociada a gramíneas en secano (Effects of the stocking rate in animal daily gains, finnish weight, costs for feeds and financial returns on finnish beef cattle operations with Leucaena 'Perú' associated with grass without irrigation).

\begin{tabular}{|c|c|c|c|c|c|}
\hline Indicadores & $1,4-1,9 \mathrm{a} / \mathrm{ha}$ & $2,0-2,4$ a/ha & E.S. & Sig & $\mathrm{CV}(\%)$ \\
\hline Raza & Cebú & Cebú & & & \\
\hline Ganancia diaria $(\mathrm{kg})$ & 0,981 & 0,749 & 0,053 & * & 11,0 \\
\hline Peso vivo final (kg) & 472 & 446 & 17,2 & * & 9,2 \\
\hline Gastos/ suplementos (\$) & 4283 & 4551 & 188 & * & 17,5 \\
\hline Ingresos/operaciones (\$) & 23114 & 15293 & 1005 & * & 19,3 \\
\hline
\end{tabular}




\begin{tabular}{|c|c|c|c|c|c|}
\hline Indicadores & Años 1 y 2 & Años del 3 al 5 & Años del 6 al 10 & ES & Sig \\
\hline Raza & Cebú & Cebú & Cebú & & \\
\hline Peso vivo final (kg) & $421^{a}$ & $416^{a}$ & $455^{b}$ & 6,1 & * \\
\hline Ganancia diaria $(\mathrm{kg})$ & $0,897^{a}$ & $0,835^{\mathrm{a}}$ & $0,971^{b}$ & 0,17 & * \\
\hline Carga (animales/ha) & 1,8 & 1,9 & 2,1 & -- & -- \\
\hline $\begin{array}{l}\text { Producción/ha/año }(\mathrm{kg}) \\
\text { Población de Leucaena (\%) } \\
\text { Población de pastos (\%) }\end{array}$ & $\begin{array}{c}1248^{a} \\
77 \\
58\end{array}$ & $\begin{array}{c}1231^{a} \\
90 \\
66\end{array}$ & $\begin{array}{c}1279^{b} \\
93 \\
71\end{array}$ & $\begin{array}{c}39,2 \\
---\end{array}$ & * \\
\hline $\begin{array}{l}\text { Ingresos por la producción } \\
\text { total }\end{array}$ & 21168 & 22050 & 22785 & -- & -- \\
\hline $\begin{array}{l}\text { Ingresos de la producción } \\
\text { por ha }\end{array}$ & 1764,1 & 1837,5 & 1898,7 & -- & -- \\
\hline Gastos totales & 16894,9 & 16263,9 & 16350,8 & -- & -- \\
\hline Costo por kg de PV & 1.60 & 1,53 & 1,50 & -- & -- \\
\hline Costo por peso producido & 0,79 & 0,73 & 0,71 & -- & -- \\
\hline Relación beneficio/costo & 1,25 & 1,35 & 1,39 & -- & -- \\
\hline
\end{tabular}

$a, b, c$ letras distintas indican diferencias significativas a $p<0,05$.

\section{BIBLIOGRAFÍA}

Cino, D.M.; Díaz, A.; Castillo, E. y Hernández, J.L. 2011 . Ceba vacuna en pastoreo con Leucaena leucocephala: algunos indicadores económicos y financieros para la toma de decisiones. Rev Cuba Cienc Agric, 45: 7-10.

Díaz, A.; Martín, P.C.; Castillo, E.; Hernández, J.L. 2012. Suplementación de añojos Charolais de Cuba en pastoreo de asociación múltiple de leguminosas herbáceas y gramíneas tropicales. Rev Cuba Cienc Agric, 46: 249-252.

Guevara, G.; Guevara, R.; Curbelo, L.; González, R.; Pedraza, R.; Martínez, S. y Estévez, J. 2009. Factores fundamentales de sostenibilidad de los sistemas de producción de ceba en fincas comerciales con bajos insumos. Informe resumen de proyecto CITMA. 14 pp.
Ku Vera, J.C.; Briceño, E.G.; Ruiz, A.; Mayo, R.; Ayala, A.J.; Aguilar, C.F.; Solorio, F.J. y Ramírez, L. 2014. Manipulación del metabolismo energético de los rumiantes en los trópicos: opciones para mejorar la producción y la calidad de la carne y leche. Rev Cuba Cienc Agric, 48: 43-53.

Milera, M. 2013. Principios para el manejo y utilización de pastos y forrajes para la producción de carne y leche en Cuba. Resumen de la obra del premio nacional de ganadería. Ministerio de la Agricultura. Cuba. 26 pp.

Pérez Infante, F. 2010. Ganadería eficiente. Editorial ACPA. La Habana. Cuba. 256 pp.

SYSTAT. 2007. The systems for statistic (Versión 11.0). SPSS Inc. Michigan. USA. 